\title{
ON THE EVOLUTIONARY STATUS AND INSTABILITY MECHA- NISM OF THE LUMINOUS BLUE VARIABLES (LBV)
}

\author{
André Maeder \\ Geneva Observatory \\ CH-1290 Sauverny, Switzerland
}

\begin{abstract}
Various evolutionary sequences leading to LBV are examined. The sequence O-OfLBV-WR-SN is well supported by the models; some LBV with relatively lower luminosity may turn into OH/IR sources. The overall duration of the LBV phase depends mainly on the average mass loss rate; for $\langle\dot{M}\rangle=10^{-3} M_{\odot} y^{-1}$, it lasts about $10^{4} \mathrm{y}$.

Very massive stars undergo, when they reach $\log T_{\text {eff }}=3.9$, strong departure from hydrostatic equilibrium due to supra-Eddington luminosities at some depth in the outer layers. This results in heavy mass loss, as the growth rate of the instability is very fast. We suggest that the amount of mass ejected in a shell episode is mainly determined by the mass of such a layer that its thermal adjustment timescale is within an order of magnitude of the stellar dynamical timescale. Simulations of B-light curves due to shell ejections by LBV are performed and some sensitive properties are identified.
\end{abstract}

\section{INTRODUCTION}

LBVs are extreme supergiants which have experienced one or several violent and large shell ejections; in the Galaxy, such typical objects are $\eta$ Car, P Cyg, AG Car and HR Car. We shall examine here what stellar models may teach us about the evolutionary sequences leading to and from the LBV stage. The structural properties will also be examined in order to find indications about the driving instability mechanism leading to the observed outbursts and about the characteristic timescales of these objects.

\section{EVOLUTION TOWARDS AND FROM THE LBV STAGE}

Several observational properties of the LBV are to be matched by the evolutionary models:

1. Their location in the HR diagram (cf. Humphreys, 1987).

2. Their $\mathrm{He}, \mathrm{C} / \mathrm{N}$ and $\mathrm{O} / \mathrm{N}$ ratios (cf. Davidson et al., 1984).

3. Their high average mass loss rates $\dot{M}$ and their episodic outbursts (cf. Lamers, 1987).

4. The existence of transition objects, like R 127 and AG Car (cf. Stahl et al. 1983; Stahl 1986).

5. Their light curve and in particular the constancy of $L$ during the variations (cf. Appenzeller and Wolf 1981).

6. Possibly, their association to young stellar groups (cf. Lortet, e.g. this meeting). 
This list is certainly non-exhaustive. These observational constraints have generally been considered to support the view that LBV are massive post-main sequence supergiants undergoing large shell ejections (cf. Humphreys, Davidson 1979; de Jager 1984; Stothers and Chin 1983; Maeder 1983; Appenzeller 1986, 1987; Lamers 1986, 1987; Humphreys 1987). The observed surface abundances in LBV are particularly decisive arguments in this context.

We have now to figure out more closely what might be the evolutionary sequence leading towards and then from the LBV, the status of which is still somehow controversial. For purpose of clarity, let us classify the evolutionary sequences of massive stars by the stage as a result of which a bare core (i.e. a WR star) is formed (cf. Chiosi and Maeder, 1986). Starting by the most massive stars, the first evolutionary sequence to be identified is the following one:

\subsection{Bare core formation during the H-burning phase}

It is possible that, as a result of heavy mass loss during the $O$ and $O f$ stages, the very massive stars loose most of their original envelope during the $\mathrm{H}$-burning phase. As a consequence, the evolutionary sequence would be

$$
O-O f-W R-S N
$$

This is indeed just the Conti scenario (1975) for the formation of WR stars; illustrations of it can be found in Maeder (1980). In view of the currently observed mass loss rates, this scenario appears feasible for initial masses above about $120 M_{\odot}$. Thus, it is possible that the most massive stars due to heavy mass loss on the MS avoid the LBV phase, a suggestion also consistent with Lortet (cf. this meeting). Any mixing mechanisms, such as overshooting, rotational or tidal diffusion, would also favour this scenario.

\subsection{Bare core after the LBV stage}

This sequence illustrated by several models with $\mathrm{M} \geq 60 M_{\odot}$ (cf. Maeder 1983; Maeder and Meynet 1987) is likely the typical one leading to the formation of LBV

$$
\mathrm{O}-\mathrm{Of}-(\mathrm{BSG})-\mathrm{LBV}-\mathrm{WR}-\mathrm{SN} \quad \sim 50-120 M_{\odot}
$$

Here, after central H-exhaustion, the star undergoes a fast increase of its radius and a rapid (in $10^{3}-10^{4} y$ ) redwards evolution in the HR diagram (cf. Fig. 1). Then it reaches an instability limit, the nature of which is discussed below (cf. $\S 3$ ) and is likely to be related to the supra- Eddington luminosity reached in some of its outer layers. As a consequence, mass loss occurs at instantaneous rates $\dot{M} \geq 10^{-2} M_{\odot} y^{-1}$. Some first results on the physical mechanism driving this ejection, the $\dot{M}$-rates, the involved timescales, the stellar models and simulations of light curves are examined in $\$ 3$ below. After an ejection, the models show a rapid blue shift, in a few years or tens of years depending on the mass loss rates in the shell ejection (cf. Fig. 2). Then the internal evolution again brings the star to the red with a recovery time, which for a given stellar mass is function of the amount of mass ejected in the shell. Keeping nearly at constant $L$, the star again reaches the instability limit corresponding to its new mass and composition and is ready for a new violent episode.

We emphasize that the properties and duration of the $L B V$ phase are essentially determined by the mass loss rates before and during the $L B V$ phase. For a typical $\langle\dot{M}\rangle=$ $10^{-3} M_{\odot} y^{-1}$ over all the LBV phase (cf. Lamers, 1986), the LBV phase may be estimated 


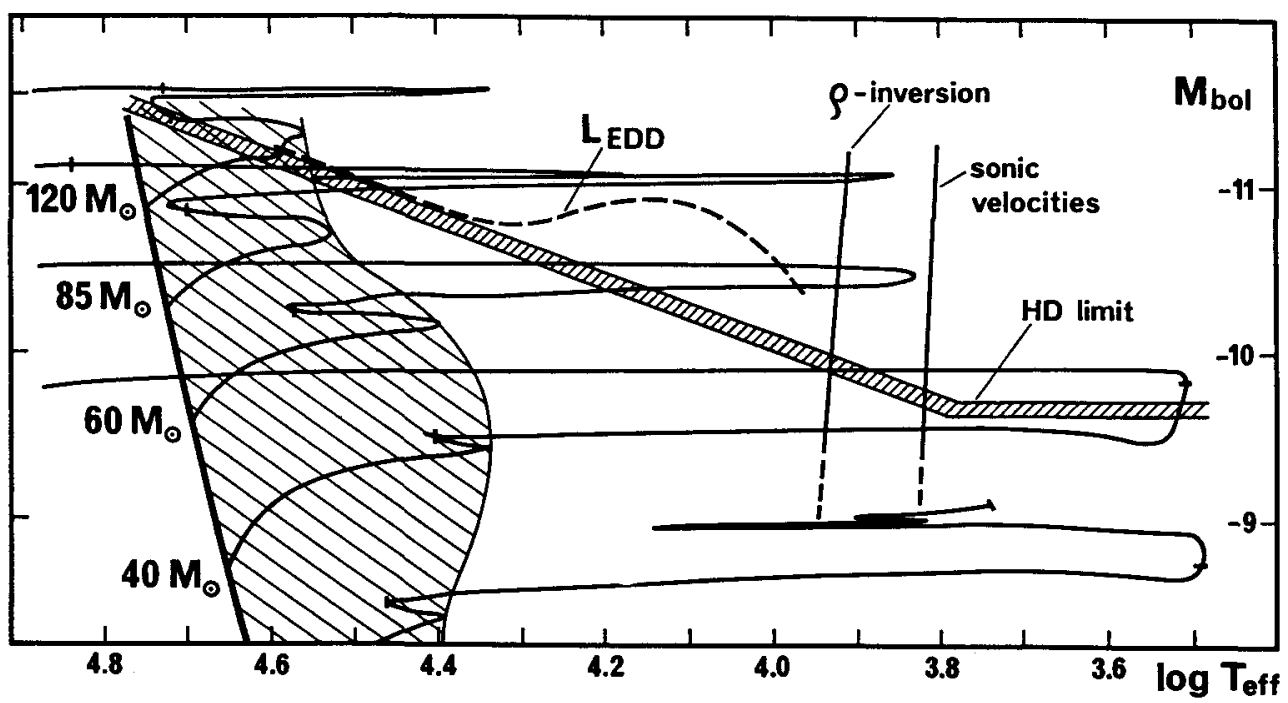

Figure 1: Evolutionary tracks in the HR diagram from Maeder and Meynet (1987), with indications of the Humphreys-Davidson limit (1979), of the limit where $L=L_{E D D}$ (cf. Lamers and Fitzpatrick, 1988), and of the locations where p-inversions and sonic velocities are met in the models.

to have a duration $t_{L B V} \leq 10^{4} y$. The argument is the following one. The amount of mass located between the surface left at the end of the MS phase and the H-burning shell is less than or of the order of $10 M_{\odot}$ (cf. Fig. 3 by Maeder and Meynet, 1987). This amount gives an upper bound to the mass to be removed in the LBV phase. Depending on $\langle\dot{M}\rangle$ in $\mathrm{LBV}$, different durations $t_{L B V}$ may therefore be obtained. Of course, the star may run into several LBV episodes. When the surface $H$-content is lower than about $X=0.3$, which corresponds to different core mass fractions according to the initial mass, the star makes its last blueward motion likely towards the WNL stage. We notice that for a given initial mass, the lifetime ratio $t_{L B V} / t_{W R}$ is of the order of 0.02 , within a factor of two at least.

Observational evidences for the sequence Of - LBV - WR are provided by the objects R 127 (Stahl et al. 1983) and AG Car (cf. Stahl 1986).

\subsection{Bare core after the stage of $\mathrm{OH} / \mathrm{IR}$ source}

The following sequence through the stage of $\mathrm{OH} / \mathrm{IR}$ source should in principle be possible :

$$
\text { O - Of - (BSG) - LBV - OH/IR - WR - SN }
$$

OH/IR sources are near the upper L limit of red supergiants, with IR excess, OH maser and optically thick dust shell (cf. Humphreys, 1987).

Indeed, if $\dot{M}<10^{-3} M_{\odot} y^{-1}$ (depending on mass), the redwards motion through the HR diagram is not stopped and the star reaches the red stage. We may speak of a "transparency of the instability limit". 
The opposite sequence of $\mathrm{OH} / \mathrm{IR}$ source evolving to LBV is not supported by our models. The stellar mass is smaller, but the opacity is also smaller, due to the much lower H-content, so that the Eddington luminosity may not be smaller. The observations of Var. A in M33 and IRC +104020 by Humphreys (1987) support the above scenario.

Mass domains for lower initial masses may of course be distinguished, but their relevance to the LBV is uncertain, although the case of SN1987A has shown that further progress is also needed in this range.

\section{PHYSICAL CONSIDERATIONS ON LBV OUTBURSTS}

\subsection{Generalities}

We shall not discuss here all the possible and impossible instabilities considered for explaining the LBV variability, since most have been ruled out (cf. Stothers and Chin 1983). Let us just mention that the recent claim that vibrational instability by the $\epsilon$-mechanism (cf. Doom et al. 1986) is responsible for the LBV mechanism cannot be supported. As stars become supergiants the ratio of central to average densities $\rho_{c} / \bar{\rho}$ grows and the stability with respect to vibrational instability increases very much (cf. Maeder 1985).

Appenzeller (1986), Lamers (1986), Lamers and Fitzpatrick (1988) have convincingly proposed that radiation pressure effects on the atmospheres cause the LBV instability. According to them the contribution of metal line opacities in the Balmer continuum considerably reduces the Eddington luminosity $L_{E D D}$. The limit they obtain for $L_{E D D}$ is drawn in Fig. 1 and it more or less coincides with the blue part of the Humphreys- Davidson limit.

\subsection{The occurrence of a catastrophe during redwards evolution}

What can be learned about the LBV instability from evolutionary models? Let us examine here some first preliminary results.

The most conspicuous effect in models of very massive stars evolving, after MS evolution, towards the red side of the HR diagram is the occurrence of a density inversion (cf. also Bisnovatyi-Kogan and Nadyozhin 1972). In our models (cf. Maeder 1987), this density inversion typically appears for stars with $\log T_{\text {eff }} \simeq 3.9$ (cf. Fig. 1) and it grows very fastly at lower $T_{\text {eff }}$. The $\rho$-inversion occurs at a distance corresponding to $10^{-4}$ to $10^{-6}$ in mass fraction from the surface; it lies in the layers where is located the opacity peak due to partial $\mathrm{H}$-ionisation and where $L$ happens to be larger than the local Eddington luminosity (account being properly given to the various opacity sources of the models). Physically, the origin of the $\rho$-inversion is the following one:

As the star expands

$$
(\rho, T) \downarrow \Rightarrow \kappa \nearrow \Rightarrow \frac{L}{L_{E D D}}>1 \Rightarrow \rho-\text { inversion }
$$

since $F_{\text {conv }} \simeq 0$.

Let us clearly emphasize that the occurrence of $\rho$-inversions in the models does not necessarily mean that $\rho$-inversions effectively occur in real stars. Rather, the occurrence of a strong $\rho$-inversion, which is a consequence of the local supra-Eddington luminosity, must be considered as the sign that hydrostatic models are no longer valid in the upper right corner of the HR diagram and that massive outflows occur. An opposite point of view was supported by Stothers and Chin (1973). However, in our opinion the absence of hydrostatic solutions in the upper right corner of the HR diagram is well indicated by the fact that 
the matching of the Henyey interior solutions with the outer solutions can progressively no longer be achieved as the star moves to the right. We may also point out that $\rho$-inversions occur in the subsonic regime of convective velocities, i.e. for $V_{\text {conv }} / V_{\text {sound }} \lesssim 0.1$; thus, the instability revealed by the $\rho$-inversion is not the same as that due to turbulent pressure and studied by de Jager (1984). Indeed, on a redwards track the $\rho$-inversions are met before sonic velocities are reached (cf. Fig. 1). The breakdown of hydrostatic solutions is due to supraEddington luminosities in the region of the opacity peak, and is thus the continuation for stars of lower $T_{\text {eff }}$ of the instability proposed by Appenzeller and Lamers (above reference) from atmospheric studies of hotter stars.

The departure of hydrostatic equilibrium revealed by the $\rho$-inversions which can reach a factor of 10 near $\log T_{\text {eff }} \simeq 3.9$, is quite large and therefore heavy mass loss is to be expected. Rates of mass loss as high as $0.5 M_{\odot} y^{-1}$ have been obtained from non-static solutions (cf. Bisnovatyi-Kogan and Nadyozhin 1972). We can roughly estimate the above figure by simple analytical arguments. Let us call $\tau_{\dot{M}}=M / \dot{M}$ the characteristic time of mass loss and $\tau_{\rho}$ the characteristic time for the growth of the $\rho$-inversion in the models. If $\tau_{\rho}<\tau_{\dot{M}}$, the non-contraried $\rho$-inversion would grow without any limit. At the opposite, $\tau_{\dot{M}}$ cannot be much shorter than $\tau_{\rho}$, since the mass loss is driven by the instability announced by the $\rho$-inversion. Thus $\tau_{\dot{M}}$ and $\tau_{\rho}$ must be of the same order of magnitude, which means

$$
\dot{M} \simeq \frac{M}{\tau_{\rho}}
$$

For an initial $60 M_{\odot}$ model in the LBV stage, we have typically $M \simeq 40 M_{\odot}$ and $\tau_{\rho} \simeq 10^{2} y$, thus we get $M$-rates of the order of magnitude of several $0.1 M_{\odot} y^{-1}$. The above value is only a rough estimate of the order of magnitude, but it tells us that the strong departure from hydrostatic equilibrium in the upper right corner of the HR diagram must result in phases of intense mass loss since the growth rate of the instability is extremely short.

\subsection{Why shell ejections?}

Central questions about $\mathrm{LBV}$ are why matter is ejected in violent outbursts rather than in the form of a steady outflow, and what determines the amount of mass in the ejected shell. This question is a critical one, which could by the way also be asked for other kinds of variable stars. Indeed, if only the matter in and above the $\rho$-inversion would be expelled in one episode, the amount of matter in the ejected shell would be at most $\sim 10^{-4}$ of the stellar mass; this is far less than observed.

Some understanding may come from considerations about the timescales involved in the problem. The timescale for thermal adjustment is very short in the outer layers of an LBV (i.e. about 70 days in an external layer of $0.1 M_{\odot}$ with the numerical data given below). Thus, during the dynamical process of ejection itself which lasts $t_{e j}$ (which typically is some multiple $\chi$ of the dynamical timescale $t_{f f}$ since matter is progressively accelerated outwards by radiation effects) the opacity peak will be able to go down within the star by as much as the local thermal timescale $t_{K H}$ allows it. For a layer with mass $\Delta m$, this local timescale is of the order of

$$
t_{K H} \simeq \frac{G M \Delta m}{R L}
$$

The $\kappa$-peak may move down during the ejection by a distance encompassing the mass $\Delta m$ fixed by the condition 


$$
\frac{G M \Delta m}{R L} \simeq t_{e j} \simeq \chi t_{f f} \simeq \chi\left(\frac{R^{3}}{G M}\right)^{1 / 2}
$$

which gives

$$
\begin{aligned}
\frac{\Delta m}{M} & \simeq \frac{\chi R^{5 / 2} L}{G^{3 / 2} M^{5 / 2}} \\
& \simeq 1.61 \cdot 10^{-12} \frac{\chi\left(R / R_{\odot}\right)^{5 / 2}\left(L / L_{\odot}\right)}{\left(M / M_{\odot}\right)^{5 / 2}}
\end{aligned}
$$

This gives an estimate of the order of magnitude of the maximum possible mass ejected by the $\kappa$-peak during some dynamical time scales.

As a numerical example let us consider a model of initially $60 M_{\odot}$ having at an age of $3.71 \cdot 10^{6} y$ an actual mass of $41.2 M_{\odot}, \log L / L_{\odot}=6.05, \log T_{\text {eff }}=3.89$ and $R=586 R_{\odot}$. This model has just a growing $\rho$-inversion with $\tau_{\rho} \simeq 10^{2} y$. For the above values we get

$$
\Delta m \simeq 0.06 \chi M_{\odot}
$$

If we take $\chi=1$ to 10 , we get $\Delta m=0.06$ to $0.6 M_{\odot}$ This amount is ejected during the time $t_{e j}$ and this implies that the mass loss rate during the ejection amounts to about the critical value of

$$
\dot{M} \simeq \frac{\Delta m}{t_{e j}}=\frac{L R}{G M}
$$

i.e. $0.75 M_{\odot} y^{-1}$ for the above model; this rate is of the same order as those previously obtained. The above discussion shows that the breakdown of the hydrostatic equilibrium in LBV may give rise to ejections of several tenths of $M_{\odot}$ at rates of about 0.1 to $1 M_{\odot} y^{-1}$. The physical reason why a large amount of matter is ejected in an outburst despite the fact that the $\kappa$-peak is very close to the surface resides in the fact that the blasting front (i.e. the $\kappa$-peak) has time to deeply move inwards during the explosion event itself (some dynamical timescales). As a consequence of the heavy mass loss the star undergoes rapid changes of physical conditions in the outer layers and it rapidly moves bluewards in the HR diagram on timescales which depend on the parameters of the ejection as illustrated by the light curves given in $\{3.4$ below. The opacity decreases in the critical layers which are then no longer characterized by large supra-Eddington luminosities, thus the catastrophic mass loss rates must soon cease. The hydrostatic solutions are again an acceptable approximation until secular evolution once more brings the star to the instability limit after a certain recovery time, as shown below.

\subsection{Response of an LBV to a large shell ejection. Theoretical light curves}

Let us now examine some consequences of massive shell ejections with parameters in the ranges suggested above. Many simulations have been performed for various choices of $\Delta m$, the mass of the ejected shell and for various $\dot{M}$-rates during the outburst. Only the consequences of the thermal response of the LBV to a mass ejection are considered here, and not 


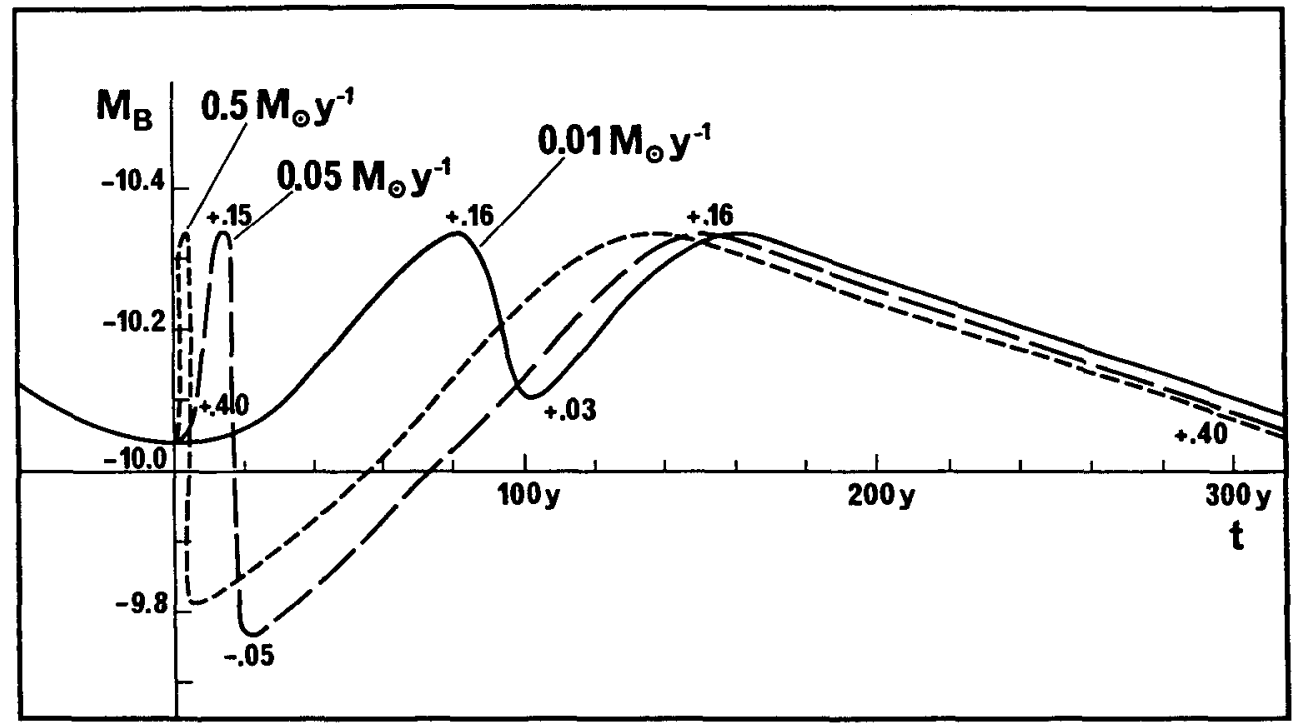

Figure 2: Example of the light curves in B-magnitude resulting from shell ejections of $1 M_{\odot}$ at various mass loss rates. Before the ejection the model had $46.9 M_{\odot}, \log L / L_{\odot}=6.04$ and $\log$ $T_{\text {eff }}=3.82$; the initial mass was $60 M_{\odot}$.

the effects of the wind and dust on the light curve; for this last aspect the reader should refer to Davidson (1987).

For ejections made at rates larger than a few $10^{-3} M_{\odot} y^{-1}$ (the exact value depends on the stellar mass), the thermal adjustment overpasses the effects of secular evolution, and the net result is a shrinkage of the stellar radius and thus a bluewards evolution in the HR diagram. The overall luminosity undergoes only limited changes. The main effect for the light curves in $B$ or $V$ magnitudes results from the change of the appropriate bolometric corrections as $T_{\text {eff }}$ varies during the horizontal oscillations in the HR diagram.

As an example and subject to the above mentioned restrictions, Fig. 2 shows three light curves for a mass ejection of $\Delta m=1 M_{\odot}$ in $10^{2} y$, in $20 y$ and in $2 y$, i.e. with $\dot{M}=0.01,0.02$ and $0.5 M_{\odot} y^{-1}$ respectively. From the many such examples calculated we can derive a few properties of LBV light curves:

1. The peak-to-peak amplitude of the $B$ or $V$ light curve is mainly a function of the total amount $\Delta m$ lost in the shell ejection: the larger $\Delta m$, the larger the amplitude (cf. Table below). From $10^{-2}$ to $1 M_{\odot} y^{-1}, \dot{M}$-rates have only a small effect (< factor 2 ).

2. The $\dot{M}$-rates during the outburst essentially determine the initial slope of the variations, i.e. the rapidity of the initial changes: the higher $\dot{M}$, the faster the initial variation (cf. Fig. 2).

3. The recovery time after an ejection, i.e. the time to again reach the same $T_{\text {eff }}$ as 
time determines the slope of the final part of the light curve after an ejection. As an example, for a $46 M_{\odot}$ star with $\log L / L_{\odot}=6.05$ and $\log T_{\text {eff }}=3.82$, one has for various cases of $\Delta m$ in the ejected shell:

\begin{tabular}{|c|c|c|}
\hline$\Delta m$ & Amplitude (B-magn) & Recovery time \\
\hline $0.3 M_{\odot}$ & 0.06 & $125 \mathrm{y}$ \\
$1.0 M_{\odot}$ & 0.51 & $350 \mathrm{y}$ \\
$3.0 M_{\odot}$ & 2.16 & $746 \mathrm{y}$ \\
\hline
\end{tabular}

4. The light curves of LBV may have very different shapes depending on $\Delta m$ and also on the $T_{\text {eff }}$ at the beginning of the ejection.

This is a first simple approach of the LBV phenomenon, but from this we see that $a$ proper analysis of the $L B V$ light curve might give an insight on the parameters of the shell ejections. Ideally, future models should be non static and should also incorporate the effect of the optically thick wind and of the dust, in order to describe more closely the totality of LBV phenomena.

\section{FINAL REMARKS ON ABUNDANCES AND MODEL CONSTRUC- TION}

Theoretical abundances in LBV have been given by Maeder (1983, 1987), Maeder and Meynet (1987). Essentially, LBV are predicted to have He-enhancements with $Y=0.4-0.7$ instead of the classical value $Y=0.28$. The $\mathrm{C} / \mathrm{N}$ ratios should be equilibrium values around 0.02 , while $0 / \mathrm{N}$ is close to but not necessarily at equilibrium with values of about 0.1 to 0.9 (see above reference). In the case of $\eta$ Car (cf. Davidson et al. 1984, 1986) the observations agree with the above values.

Since the occurrence of large density inversions suggest non static solutions, does it mean that in calculations of stellar models we cannot go through the LBV phase in the framework of hydrostatic code? If no special care is taken, the models certainly break down as many have experienced it. However, an appropriate and physically consistent treatment of the outer layers (cf. Maeder 1987) may be devised to properly handle these stages even in the framework of classical stellar codes.

\section{REFERENCES}

Appenzeller, I.: 1986, in Luminous stars and associations in galaxies, IAU Symp. 116, Ed.

C. de Loore and A.J. Willis, p. 139

Appenzeller, I.: 1987, in Instabilities in luminous early type stars, Ed. H. Lamers and C. de Loore, Reidel Publ. Co., p. 55

Appenzeller, I., Wolf, B.: 1981, in The most massive stars, ESO workshop, Ed. S. D'Odorico, D. Baade and K. Kjär, p. 131

Bisnovatyi-Kogan, G.S., Nadyozhin, D.K.: 1972, Astrophys. Space Sci. 15, 353 
Chiosi, C., Maeder, A.: 1986, Ann. Rev. Astron. Astrophys. 24, 329

Conti, P.S.: 1975, Mém. Soc. Roy. Sci. Liège, 6ème série, tome IX, p. 193

Davidson, K.: 1987, Astrophys. J. 317, 760

Davidson, K., Dufour, R.J., Walborn, N.R., Gull, T.R.: 1984, in Observational tests of the stellar evolution theory, Ed. A. Maeder and A. Renzini, Reidel Publ. Co. p. 261

Davidson, K., Dufour, R.J., Walborn, N.R., Gull, T.R.: 1986, Astrophys. J. 305, 867

Doom, C., de Greve, J.P., de Loore, C.: 1986, Astrophys. J. 303, 136

Humphreys, R.M.: 1987, in Instabilities in luminous early type stars, Ed. H. Lamers and C. de Loore, Reidel Publ. Co. p. 1

Humphreys, R., Davidson, M.K.: 1979, Astrophys. J. 232, 409

de Jager, C.: 1984, Astron. Astrophys. 138, 246

Lamers, H.: 1986, in Luminous stars and associations in galaxies, IAU Symp. 116, Ed. C. de Loore and A.J. Willis, Reidel Publ. Co. p. 157

Lamers, H.: 1987, in Instabilities in luminous early type stars, Ed. H. Lamers and C. de Loore, Reidel Publ. Co. p. 99

Lamers, H., Fitzpatrick, E.L.: 1988, Astrophys. J. 324, 279

Maeder, A.: 1980, Astron. Astrophys.92, 101

Maeder, A.: 1983, Astron. Astrophys.120, 113

Maeder, A.: 1985, Astron. Astrophys.147, 300

Maeder, A.: 1987, Astron. Astrophys.173, 287

Maeder, A., Meynet, G.: 1987, Astron. Astrophys.182, 243

Stothers, R., Chin, C.W.: 1973, Astrophys. J.179, 555

Stothers, R.N., Chin, C.W.: 1983, Astrophys. J.264, 583

Stahl, O.: 1986, Astron. Astrophys.164, 321

Stahl, O., Wolf, B., Klare, G., Cassatella, J., Krautter, J., Persi, P., Ferrari-Toniolo, M.: 1983, Astron. Astrophys.127, 49

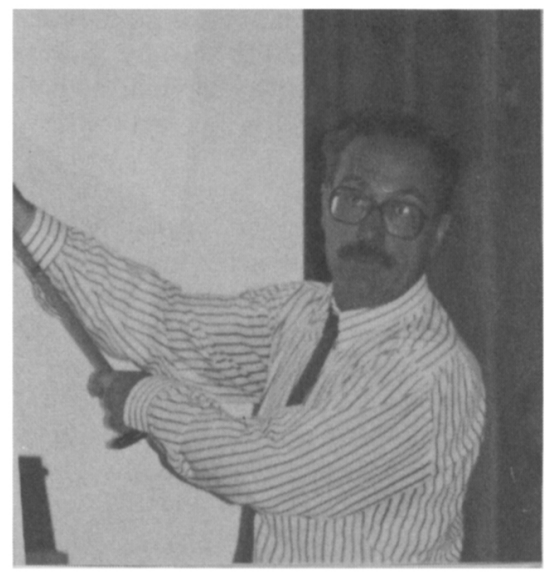

Andre Maeder 


\section{DISCUSSION}

Owocki: Is it correct to view the density inversion as arising when the luminosity exceeds the Eddington limit, yielding a net outward effective gravity, and thereby requiring a positive pressure (and hence density) gradient? Have you attempted to model the time-dependent mass loss that this density inversion implies? What are the expected flow speeds of ejecta? Can they be supersonic? What determines how much mass is ejected?

Maeder: The answer to your first question is yes. The actual luminosity exceeds the Eddington limit in the region of the opacity peak and this causes the density inversion in hydrostatic calculations. However, I do not expect the same in non-static models, where very likely an outward motion would occur instead. What I have modeled is essentially the star's thermal response to shell ejections having a variety of parameters, in order to see how the resulting light curves can give information on the masses of ejected shells and on the rates of mass loss during ejections.

The depth of the layers where the density inversion occurs is certainly a critical parameter determining the amount of mass ejected. However, this critical level may move downward during the shell ejection itself, by an amount which is fixed by the condition that the local thermal timescale should not become longer than the duration of the shell ejection. Thus, I expect the amount of mass in a shell ejection to be a multiple of the mass above the density inversion.

Langer: Some comments on your proposition that the density inversion in supergiant envelopes may be responsible for violent shell ejections:

(1) The density inversion may be avoided by taking the density scale height instead of the pressure scale height in the mixing length theory. (2) Even if a density inversion develops, the Rayleigh-Taylor instability will sweep it out. (3) How can a turbulent process like the Rayleigh-Taylor instability lead to outward-directed forces and subsequent shell ejections?

Maeder: The density inversion can be avoided by some artifices, such as multiplying the pressure scale height by 10 or more. Although I consider that static models are not appropriate for very luminous red supergiants, what I showed is that by a proper analysis of the Rayleigh-Taylor instability we can find a better way of handling the problem in the framework of static models than by just artificially increasing the mixing-length ratio.

Sreenivasan: Semi-convection zones tend to appear in our models for $\log T_{\text {eff }}$ between 3.9 and 3.7 or so. These can effectively smooth out any such density inversions. The semi-convection zones appear far above the core just as you find for the density inversion. There is not much mass loss between these points on the evolution track. The CAK-type mass loss has switched itself off and there is not yet an extensive convection zone to produce a real giant-type wind driven by mechanisms analogous to the solar wind. A Rayleigh-Taylor instability should also smooth out density inversions but it does not, so far as I know, produce abrupt mass ejections. So I really do not understand what you are saying. Could you explain it further please?

Maeder: As is well known (cf. Chiosi and Maeder in Ann. Revs. Astron. Astrophys., 1986), mass loss and convective overshooting can make the semi-convection zones essentially disappear in massive stars (except for initial masses around 12 to $15 \mathrm{M}_{\odot}$, well below the LBV range). The statement $I$ made is that the density inversion frequently found in static models of stars evolving toward extremely luminous red supergiants (above and near the H-D limit) is just the sign that static models are 
no longer valid for these stars and that an instability with heavy mass loss is encountered. Thus there is no question of the Rayleigh-Taylor instability itself driving the ejection.

Lamers: Your mechanism related to density inversion occurs around $T_{\text {eff }} \approx 9000 \mathrm{~K}$; but the large LBV outbursts are observed to occur when $T_{\text {eff }} \approx 20000$ to $30000 \mathrm{~K}$.

Maeder: The instability effectively occurs at about $\log T_{\text {eff }} \approx 3.9$; then the star very rapidly moves to a much higher $T_{\text {eff }}$, the value of which depends on the amount of gas ejected. The star model spends relatively much more time at this higher $T_{\text {eff }}$. I agree that whether this behavior corresponds to the observed one, or not, has to be carefully verified.

Kahn: Do similar effects occur also for stars with higher surface temperatures, where the density inversion demanded by a static model is smaller, and if so do they lead to a less violent dynamic response?

Maeder: Of course, before being large, the density inversion starts small. Its growth rate is quite short, though (a few times $10 \mathrm{y}$ ), which implies that the initially small instability is likely to become a larger one soon.

De Groot: You showed a viewgraph with light curves for three different mass-loss rates during an outburst, and identified one of these with $\eta$ Carinae. However, while the light curve looks very much like that of $\eta$ Car, you also said that dust was not included in your calculation. Since dust is crucial for the light curve of $\eta$ Car, I do not see the relevance of the curve that you showed. On the other hand, your next viewgraph showed a light curve that very much resembles the historical light curve of P Cygni; so maybe you do have a point in all this.

Maeder: The simulated light curves are just illustrations of the thermal responses of LBV stars for various choices of the mass of the ejected shell and for various choices of the mass-loss rates during the episode of shell ejection.

Baratta: What is the mass-loss rate in the case of the more massive stars, which may avoid the LBV phase?

Maeder: I have given some numerical values for quasi-homogeneous evolution in a 1980 paper, Astr.\& Astrophys. 92, 101. Essentially, the observed rates allow this scheme for masses above $100 \mathrm{M} \odot$.

Van der Hucht: In their original paper (1972), Bisnovatji-Kogan and Nadyazkin related the occurence of the density-inversion-induced shell ejection to the presence of ring nebulae around some WR stars. Can it be that WR stars with "ejecta-type" ring nebulae are post-LBV objects?

Maeder: This is an interesting suggestion. In order to verify it, more data on the masses and compositions of the ring nebulae are necessary.

Conti: With O-type stars we have to keep in mind that the main sequence, the core H-burning phase, may well include stars that are classified as supergiants. The total mass loss during the main sequence phase is a product of the mass-loss rate(s) and the total lifetime. Could LBV's be H-burning objects?

Maeder: I would not expect LBV stars to be in the core $\mathrm{H}$-burning phase but more likely in the shell $\mathrm{H}$-burning or possibly at the very beginning of the central $\mathrm{He}$ burning phase. 
De Jager: Van Genderen monitored some hundred super- and hyper-giants in a recent survey. Among a considerable number of LBV's, he found that their short-time-scale variations have longer "quasi-periods" (or "semi-periods") than non-LBV supergiants in the same part of the H-R diagram. With period $\sim \rho^{-1 / 2}$, this means that LBV's have smaller masses than supergiants of the same $L$ and $T_{\text {eff. Hence, LBV's are on }}$ the blueward track of evolution.

Maeder: LBV's are likely on tracks evolving back and forth in the H-R diagram and whether they are moving blueward or redward is difficult to determine. But I agree with your conclusion that the longer periods may be consequences of lower actual mass for the LBV stars, in or between successive shell ejections -- provided, of course, that the nature of the pulsations and the pulsation modes are the same for both categories of stars observed. 